\title{
Transferable organic semiconductor nanosheets for application in electronic devices
}

Simon J. Noever, Michael Eder, Fabio del Giudice, Jan Martin, Franz Werkmeister, Stefan

Hallwig, Stefan Fischer, Oliver Seeck, Nils-Eike Webert, Clemens Liewald, Fritz Keilmann, Andrey Turchanin* and Bert Nickel*

Simon J. Noever, Michael Eder, Stefan Fischer, Fabio del Giudice, Jan Martin, Stefan Hallwig, Clemens Liewald, Franz Werkmeister, Dr. Fritz Keilmann, PD. Dr. Bert Nickel

E-mail: nickel@physik.uni-muenchen.de

Faculty of Physics and CeNS, Ludwig-Maximilians-University, 80539 Munich, Germany

Simon J. Noever, Clemens Liewald, PD. Dr. Bert Nickel

Nanosystems Initiative Munich (NIM), 80799 Munich, Germany

Dr. Oliver Seeck

Deutsches Elektronen Synchrotron DESY, 22603 Hamburg, Germany

Dr. Nils-Eike Weber

Faculty of Physics, University of Bielefeld, 33615 Bielefeld, Germany

Present Addresses: $\uparrow$ Scienta Omicron GmbH, 65232 Taunusstein, Germany

Prof. Dr. Andrey Turchanin

Institute of Physical Chemistry, Friedrich Schiller Jena University, 07743Jena, Germany

Jena Center for Soft Matter (JCSM), 07743 Jena, Germany

Center for Energy and Environmental Chemistry (CEEC), 07743 Jena, Germany

E-mail: andrey.turchanin@uni-jena.de 


\begin{abstract}
(for reviewing purposes):
We have developed a method to stabilize and transfer nanofilms of functional organic semiconductors. The method is based on crosslinking of their topmost layers by low energy electron irradiation. The films can then be detached from their original substrates and subsequently deposited onto new solid or holey substrates retaining their structural integrity. Grazing incidence X-ray diffraction, X-ray specular reflectivity and UV-VIS spectroscopy measurements reveal that the electron irradiation of ca. $50 \mathrm{~nm}$ thick pentacene films results in crosslinking of their only topmost ca. $5 \mathrm{~nm}$ ( 3 to 4 monolayers), whereas the deeper pentacene layers preserve their pristine crystallinity. We studied the electronic performance of the transferred pentacene nanosheets in bottom contact field-effect devices and found that they are fully functional and demonstrate superior charge injection properties in comparison to the pentacene films directly grown on the contact structures by vapor deposition. The new approach paves the way to integration of the organic semiconductor nanofilms on substrates unfavorable for their direct growth as well as to their implementation in hybrid devices with unusual geometries, e.g., in devices incorporating free-standing sheets.
\end{abstract}

Keywords: organic semiconductors, 2D materials, pentacene, nanosheets, electronic devices

\title{
TOC figure
}

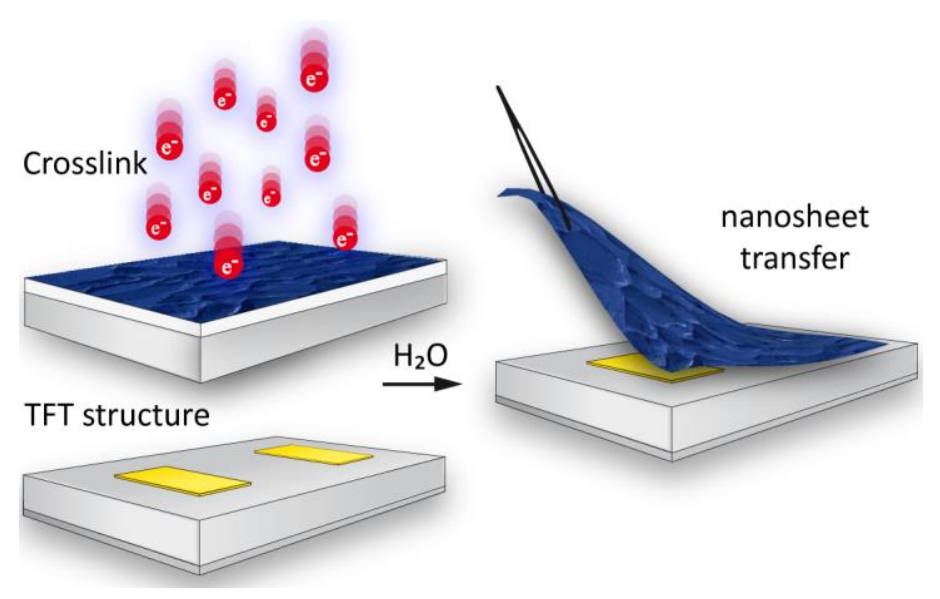


The transfer and microfabrication techniques of graphene and other two-dimensional (2D) materials have revolutionized the fabrication of novel layered materials and their implementation in electronic, optoelectronic and nanoelectromechanical devices. ${ }^{[1-5]}$ By mechanical stacking of various atomically thin sheets (e.g. graphene, $\mathrm{MoS}_{2}$, or $\mathrm{BN}$ ), novel van der Waals (vdW) heterostructures are engineered, even if these material combinations cannot be grown directly by physical vapor deposition techniques. ${ }^{[6]}$ In this way vdW heterostructures with tailored electronic and optoelectronic properties can be generated by combining metallic, insulating and semiconducting sheets. ${ }^{[7]}$ Nanofilms of organic semiconductors are promising candidates to extend this material toolbox for building hybrid devices, which would profit from the physical properties of both inorganic and organic materials. ${ }^{[8,9]}$ To this end, they have to be prepared in the form of mechanically stable and transferable sheets. However, in contrast to graphene, where atoms are linked via strong covalent bonds, small aromatic molecules in pristine organic semiconductor films are bound via weak vdW forces. Therefore, it is not possible to peel off and deposit organic films to create electronic devices, in contrast to thicker organic crystals, which have been transferred successfully. ${ }^{[10]}$ Here we demonstrate the preparation and implementation in field effect transistors (FETs) of transferable pentacene nanosheets, stabilized via electron irradiation induced crosslinking of their surface layers. The irradiated films possess high mechanical stability and therefore they can be removed from the growth substrate and transferred onto new solid substrates or suspended across macroscopic cavities and grids as freestanding structures. We characterize in detail the effect of the electron irradiation on structural and optical properties of pentacene nanofilms employing grazing incidence X-ray diffraction (GIXD), X-ray reflectometry (XR), UV-VIS and IR spectroscopy, helium ion, atomic force and scanning near field microscopy (HIM, AFM and SNOM). The functional electronic properties of the formed 
nanosheets are studied via electric transport measurements of the FET devices. We found that already about $5 \mathrm{~nm}$ (ca. 3-4 molecular layers) of crosslinking depth are sufficient to stabilize 50 $\mathrm{nm}$ thick pentacene films, whereas the remaining film preserves its pristine structure as well as electronic and optical properties and can be used for functional applications. The transferred pentacene nanosheets show superior charge injection characteristics in the FET devices in comparison to the pentacene films prepared by physical vapor deposition.

Low energy electron irradiation of aromatic self-assembled monolayers (SAMs) results in their lateral crosslinking and conversion into 2D carbon sheets - carbon nanomembranes (CNMs) with a thickness of only one molecule. ${ }^{[1]}$ The crosslinking is driven by primary electron irradiation as well as low energy secondary and photoelectrons produced in the substrate resulting in the dissociation of $\mathrm{C}-\mathrm{H}$ bonds and subsequent formation of the new covalently bonded carbon network. ${ }^{[12]}$ Similar to graphene, fully crosslinked CNMs can be removed from their substrates and transferred onto new holey or solid substrates or stacked into vdW heterostructures as free-standing sheets. ${ }^{[13,14]}$ As the penetration depth of low energy electrons can be precisely tuned in the range of a few nanometers, ${ }^{[15]}$ we employ this effect to crosslink only the topmost layers of a $\sim 50 \mathrm{~nm}$ thick film of pentacene preserving the pristine structure of the deeper layers. In the following, we present the structural and functional characterization of the surface stabilized free-standing pentacene nanosheets prepared in this way.

First, we describe the essential steps to crosslink and transfer pentacene nanosheets, Fig. 1a. We use a thin sacrificial polyvinyl-alcohol (PVA) layer deposited on a flat and inert substrate such as an oxidized Si wafer or a fused silica glass by spin coating and deposit a $50 \mathrm{~nm}$ pentacene film on this substrate by vacuum vapor deposition. ${ }^{[16]}$ The deposited film is then irradiated in the same vacuum chamber with a defocused electron beam having an electron energy of $\mathrm{E}_{\text {irr }}=500$ 
$\mathrm{eV}$ and an irradiation dose of $\mathrm{D}_{\text {irr }}=3.0 \mathrm{mC} / \mathrm{cm}^{2}$. To detach the pentacene film from the surface, the sample is removed from the vacuum chamber and immersed in deionized water. Since PVA is a water soluble polymer, the sacrificial PVA layer dissolves and the pentacene film remains free-floating in the solvent. ${ }^{[17,18]}$ These freestanding films with areas up to a size of $1 \mathrm{~cm}^{2}$ can then be picked up with tweezes, placed onto new substrates and removed from the water. The crosslinking step is essential for this procedure because pristine (non-irradiated) pentacene films disintegrate at the slightest touch by tweezers or during transfer through the water surface meniscus. After removing the wet nanosheet from water, it is possible to correct its position on the surface and flatten it out by gentle pulling. Upon drying in nitrogen flow, the nanosheets laminate firmly to the new surface, i.e. they adhere irreversibly by van der Waals forces. The HIM image in Fig. 1b shows a pentacene nanosheet, which was transferred in this way on an oxidized silicon wafer. Folds and wrinkles are recognized indicating the sheet character of the pentacene. The characteristic terrace-like topography of pentacene is conserved after irradiation and transfer (cf. supporting information (SI) Fig. S1). In Fig. 1c and Fig. 1d optical microscopy images of pentacene nanosheets spanning over holes with diameters of several millimeters are presented. The sheets are homogenous with some color variations originating from the wrinkle and folds due to transfer. In Fig. 1c a $50 \mathrm{~nm}$ pentacene nanosheet spans a $2 \mathrm{~mm}$ diameter hole in a brass sheet, whereas in Fig. 1d the nanosheet spans a TEM grid with a mesh width of $300 \mu \mathrm{m}$. As we show in the following, this remarkable mechanical stability results from the lateral crosslinking of only the topmost 3-4 monolayers (ML) of the pentacene films.

To analyze the penetration depth of the crosslinking, we employ specular XR in combination with GIXD measurements. ${ }^{[19]}$ As seen from the XR data presented in Fig. 2, the pristine film shows the characteristic $\left(\begin{array}{lll}0 & 0 & \mathrm{~L}\end{array}\right)$ reflections of the pentacene thin film phase (black curve $) .{ }^{[20]}$ 
After irradiation, the $\left(\begin{array}{lll}0 & 0 & \mathrm{~L}\end{array}\right)$ peaks broaden and decrease in intensity (red curve). This observation indicates a reduction of the crystallinity due to the electron irradiation induced crosslinking. In comparison to XR, where the total thickness of the film contributes to the signal, in GIXD an evanescent X-ray wave selectively probes only the surface region (cf. Fig. 2b). Experimentally, the probing depth is adjusted using the X-ray beam at sub-critical incidence angle for total reflection, typically at less than a fraction of a degree from the surface. The smaller the angle, the more surface sensitive is the measurement. GIXD measurements for a pristine pentacene film and films irradiated at two different electron beam energies $\left(E_{\text {irr }}=300 \mathrm{eV}\right.$ and $800 \mathrm{eV}, \mathrm{D}_{\text {irr }}=3.0 \mathrm{mC} / \mathrm{cm}^{2}$, see SI Table 1 for details) are shown in Fig. 2c. While there is still some GIXD intensity of the first truncation rod $\left(\begin{array}{lll}1 & 1 & \mathrm{~L}\end{array}\right)$ after $300 \mathrm{eV}$ irradiation, the signal vanishes almost completely after $800 \mathrm{eV}$ irradiation. To quantify the number of disordered crosslinked layers, i.e. the number of layers on top of the film which do not contribute to the diffraction signal, we measure the GIXD signal under different incidence angles and model the diffracted intensities within the model of depth controlled grazing incidence diffraction (DCGID) (Fig. 2c). ${ }^{[21-24]}$ Details on the fit routine are reported in the SI and in Fig. S2. We find that the observed intensities are in agreement with three disordered monolayers (ML) of pentacene (or $4.5 \mathrm{~nm}$ crosslinking depth) for $300 \mathrm{eV}$ irradiation and five disordered monolayers pentacene (or $7.5 \mathrm{~nm}$ crosslinking depth) for the $800 \mathrm{eV}$ sample. These data unambiguously show that only the topmost layers of the pentacene film lose their crystallinity due to the crosslinking, whereas the pristine crystallinity is preserved in the deeper layers of the film.

To get an insight into the crosslinking mechanisms, we applied Fourier transform infrared (FTIR) spectroscopy (FTIR). As seen from the FTIR spectra (cf. Fig. 3a), after irradiation the characteristic C-H vibrations of pentacene at $910 \mathrm{~cm}^{-1}$ and $733 \mathrm{~cm}^{-1}$ are strongly diminished. ${ }^{[25]}$ 
Such a behavior is indicative for the hydrogen abstraction via the cleavage of the $\mathrm{C}-\mathrm{H}$ bonds and formation of new carbon bonds between the unsaturated adjacent aromatic moieties. ${ }^{[12]}$ Next, we used UV-VIS spectroscopy to characterize the changes in the optical spectra. As seen from Fig. $3 \mathrm{~b}$, the UV-VIS spectrum of a pristine pentacene film shows the characteristic absorption features in the spectral range of 500 to $700 \mathrm{~nm} \cdot{ }^{[25]}$ After electron irradiation with two different electron energies $(500 \mathrm{eV}$ and $1 \mathrm{keV})$, the intensity of these characteristic absorbance features is decreased. This effect is stronger for the irradiation with higher energy electrons, which is in agreement with their higher penetration depth and therefore the formation of a thicker crosslinked layer. Using the Lambert-Beer law and the corresponding inelastic mean free paths of $500 \mathrm{eV}$ and $1 \mathrm{keV}$ electrons, we estimate the thickness of the formed crosslinked layer to about $3 \mathrm{~nm}$ and $17 \mathrm{~nm}$, respectively. The formation of the crosslinked layer is also in agreement with an increase of the intensity in the spectral range at smaller wave lengths (cf. Fig. 3b), which is characteristic for the formation of amorphous carbon species. ${ }^{[26]}$ To summarize the structural and optical study, we conclude that irradiation of pentacene films with electrons in the range of 300 to $500 \mathrm{eV}$ results in the crosslinking of their topmost 3-4 MLs. These topmost layers have disordered most probably amorphous structure, whereas the deeper pentacene layers preserve their pristine crystallinity. Importantly, the formed crosslinked layer provides a sufficient mechanical stability to about $50 \mathrm{~nm}$ thick pentacene films in order to transfer them onto new solid and holey substrates as free-standing sheets nanosheets.

In the following, we demonstrate that the formed pentacene nanosheets possess the functional semiconducting properties and can be employed in effective field effect devices. To this end, we fabricated bottom-contact, bottom-gate pentacene FETs by vapor deposition of pentacene in vacuum onto the contact structures and studied electric transport properties of these devices 
before and after the irradiation, Fig. 4a. After an irradiation with low energy electrons $\left(E_{\text {irr }}=350\right.$ $\mathrm{eV}, \mathrm{D}_{\text {irr }}=1.5 \mathrm{mC} / \mathrm{cm}^{2}$ ) no significant alteration in the device performance in comparison to the non-irradiated devices is observed (Fig. 4a). This demonstrates that the crosslinked layer does not penetrate into the conduction channel, which is typically confined at the semiconductordielectric interface (Fig. 4b). ${ }^{[27]}$ Only after extensive irradiation at higher electron energies and doses $\left(E_{\text {irr }}=700 \mathrm{eV}, D_{\text {irr }}=4.5 \mathrm{mC} / \mathrm{cm}^{2}\right)$ the device performance significantly decreases. In the next step, we transferred the pentacene nanosheets onto prefabricated transistor contact pads of varying bottom-contacts with channel widths of $\mathrm{W}_{c h}=10 \mathrm{~mm}$ and channel lengths of $\mathrm{L}_{\mathrm{ch}}=5$ $\mu \mathrm{m}, 10 \mu \mathrm{m}$ and $20 \mu \mathrm{m}$ (see experimental section) and compared the device performance with transistor structures prepared via conventional physical vapor deposition. The AFM images of the contact regions in Fig. $4 \mathrm{~b}$ show the morphology on both types devices. The typical obstructed pentacene growth (i.e. small grained, pillar-like 3D morphology) is observed by direct vapor deposition on gold contacts. ${ }^{[28]}$ In contrast, the structure of the pentacene films grown on PVA and transferred after the crosslinking onto gold contacts is significantly more homogeneous and shows the characteristic Bragg peaks of the pentacene thin film phase (Fig. S3). The obstructed growth of pentacene on gold has a negative influence on the charge injection properties of pentacene devices and can only be reduced by a proper, often aggressive modification of the gold contacts. ${ }^{[28-32]}$ On the other hand, the elongated pentacene structures formed on PVA most likely represent the lying phase pentacene ${ }^{[33]}$ which we expect to improve the contact resistances and therefore charge injection properties of the devices. Fig. 4c shows the calculated contact resistances for devices with different channel lengths. The data were obtained by extrapolation of their total resistance to zero channel length. ${ }^{[34,35]}$ For a gate voltage of $\mathrm{V}_{\mathrm{G}}=15 \mathrm{~V}$ the devices made of the transferred pentacene sheets have a contact resistance of $R_{p}=218 \mathrm{kOhm}$. This is 
almost two orders of magnitude less than the contact resistance of the transistors produced by the conventional pentacene vapor deposition on the test pads, which was $R_{p}=15 \mathrm{MOhm}$. This improvement is even more significant for larger gate voltages. Thus for $\mathrm{V}_{\mathrm{G}}=30 \mathrm{~V}$ the contact resistance of the devices made out for the transferred sheets is $57 \mathrm{kOhm}$ vs. $10 \mathrm{MOhm}$ for devices prepared by vapor deposition. These findings demonstrate that the presented fabrication and transfer technique of pentacene nanosheets enables the fabrication of fully functional electronic devices with superior contact characteristics and an unaffected mobility (here $\mu \sim 0.1$ $\mathrm{cm} 2 /$ Vs, cf. Fig. S4) in comparison to devices made by conventional vapor deposition.

In summary, we have introduced a novel methodology to fabricate transferrable nanosheets of organic semiconductors with few tens of nanometers thickness via irradiation of their thin films with low energy electrons. The electron irradiation results in crosslinking of the topmost molecular layers, stabilizing the whole film and enabling its transfer as a nanosheet onto new substrates. Because of the low penetration depth of the crosslinking, the studied pentacene nanosheets preserve their functional semiconducting and optical properties. Moreover, employed in bottom contact FETs, they show a reduced contact resistance in comparison to devices fabricated via direct vapor deposition on the gold electrodes. The proposed methodology opens up new possibilities towards the fabrication of organic semiconductor devices with transferable organic semiconductor nanosheets from a variety of aromatic molecules. It paves the way towards free-standing organic filed effect devices, an area which was reserved for single crystals so far, as well as to their integration with other $2 \mathrm{D}$ materials in hybrid devices. 


\section{Experimental}

The defocused electron beam was produced using a Perkin Elmer low energy electron diffraction (LEED) gun (PHI Model 11-020 LEED Electronics System). Most experiments have been performed using an electron gun emission current of $5 \mathrm{~mA}$.

The doses $\left(\mathrm{mC} / \mathrm{cm}^{2}\right)$ were estimated via the measured beam current $\mathrm{I}_{\mathrm{B}}$ flowing through a ground

electrode: $D=\frac{I_{B} t}{A}$. For the used setup and an emission current of $I_{\mathrm{em}}=5 \mathrm{~mA}$, the current through an irradiated reference surface $A=4 \mathrm{~mm}^{2}$ at the sample position was approximately $I_{B}=100 \mathrm{nA}$. Some experiments have also been performed with a reduced emission current of $2 \mathrm{~mA}$ after filament exchange.

Further details for sample preparation and characterization are reported in the supporting information. 
a)

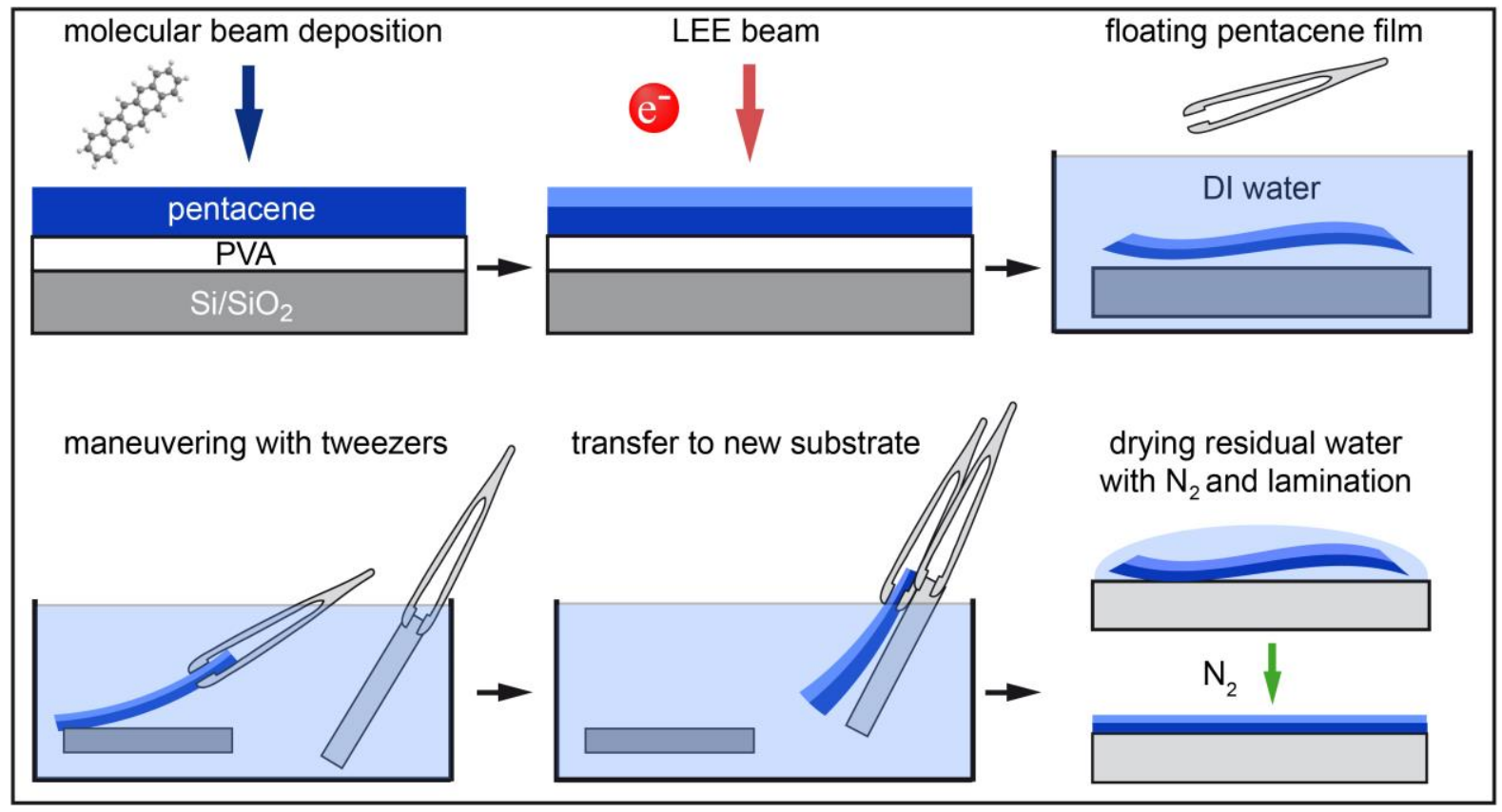

b)

c)
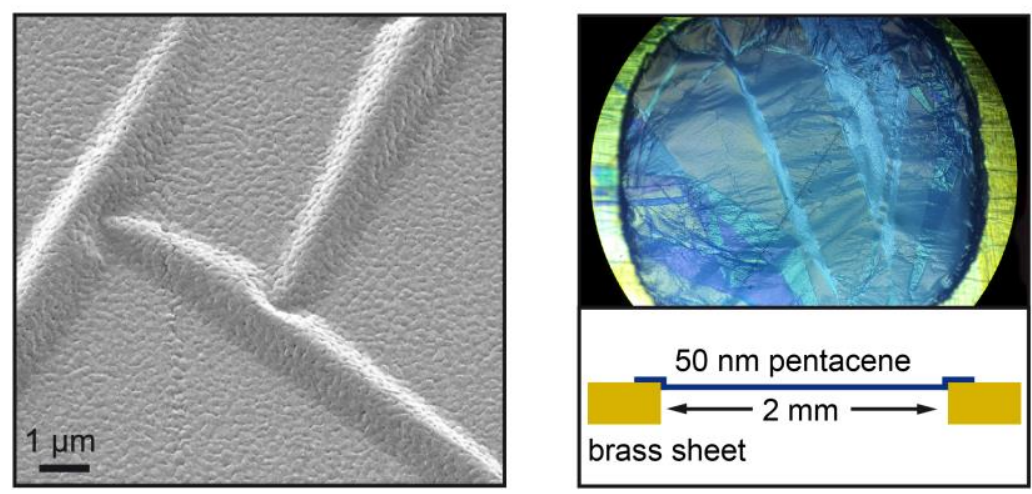

d)

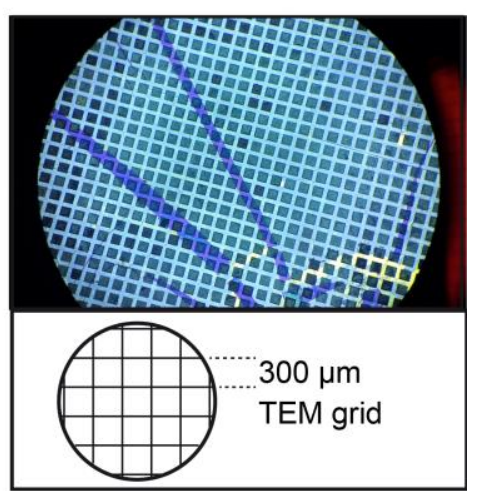

Fig. 1: Transfer of thin pentacene films. a) Schematic representation of the transfer method. b) Helium ion microscopy (HIM) image of a crosslinked and transferred $20 \mathrm{~nm}$ pentacene film. c) Optical microscopy image of a $50 \mathrm{~nm}$ thin pentacene film spanned over a $2 \mathrm{~mm}$ hole in a $0.5 \mathrm{~mm}$ brass sheet. d) Optical microscopy image of a $50 \mathrm{~nm}$ thin pentacene film transferred onto a copper TEM grid (mesh width: $300 \mu \mathrm{m})$. 
a)

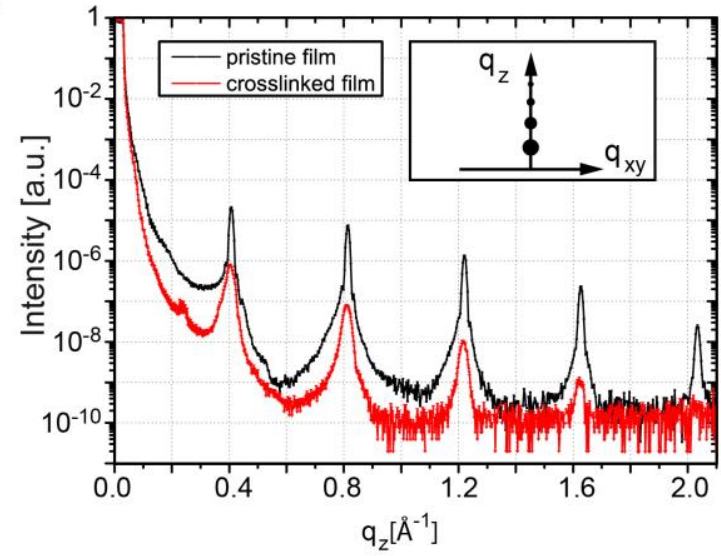

b)

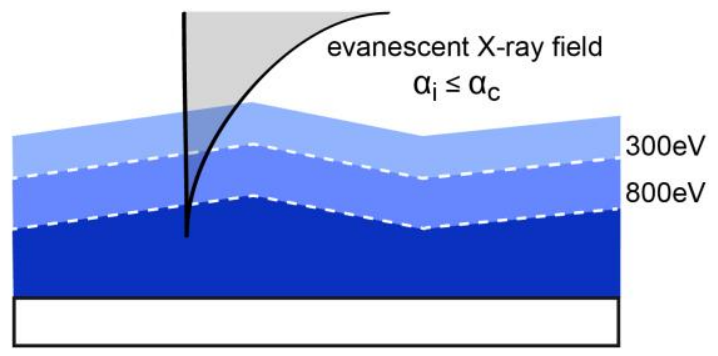

c)
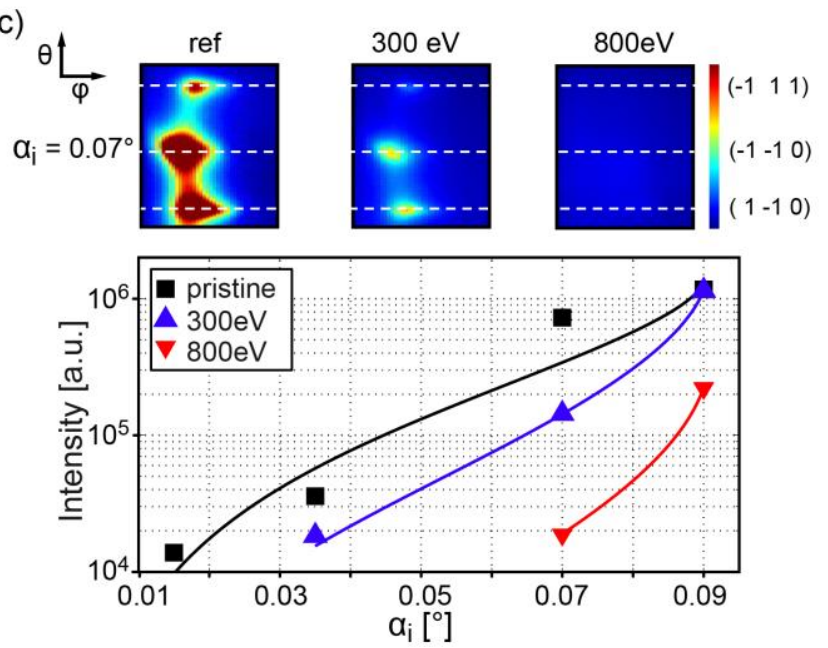

Fig. 2: X-ray analysis of the influence of crosslinking on the crystal structure of pentacene thin films. a) Comparison of synchrotron specular X-ray reflectometry measurements of a pristine pentacene thin film (black) on $\mathrm{SiO}_{2}$ and a strongly crosslinked film (red, 50 minutes at electron energy $\left.>700 \mathrm{eV}, \mathrm{D}=7.5 \mathrm{mC} / \mathrm{cm}^{2}\right)$. The decreasing Bragg signal indicates that the crosslinked film contains less crystalline material. b) Schematic of the evanescent X-ray field penetrating the 
pentacene surface for different electron irradiation depths. c) GIXD measurements of the first pentacene truncation rod at different angles of incidence and different e-beam energies (here, $\theta$ is the out-of-plane angle and $\varphi$ the in-plane angle). Top: exemplary GIXD data for pristine, $300 \mathrm{eV}$ irradiated, and $800 \mathrm{eV}$ irradiated pentacene at $\alpha_{\mathrm{i}}=0.07^{\circ}$. Bottom: the highest intensities of the (1-10) peaks (middle peaks from raw data), plotted against the angles of incidence. The continuous lines represent the fit calculated from the DCGID model for truncation rods, including non crystalline top layers. 

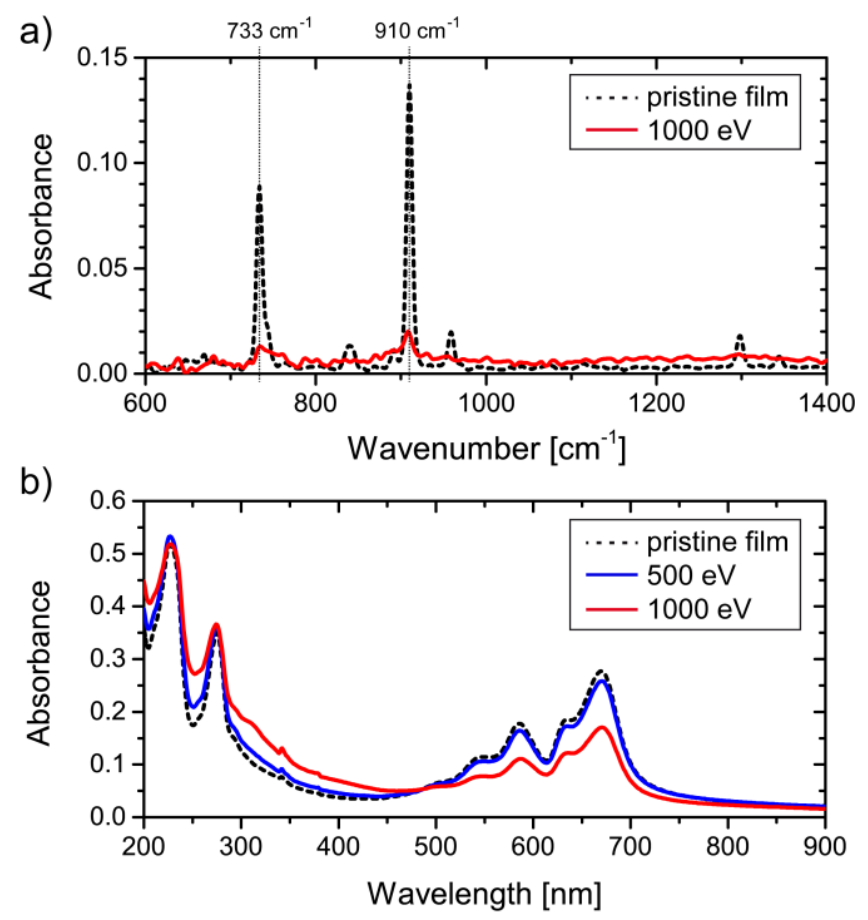

Fig. 3: Spectroscopic characterization of the crosslinking process. (a) FTIR spectra of a $23 \mathrm{~nm}$ pentacene film on $\mathrm{Au}$ before and after irradiation with $1 \mathrm{keV}$ electrons, shown as dashed black and red curve, respectively. The electron gun emission current was $2 \mathrm{~mA}$, and irradiation time was 20 minutes in all cases. (b) UV-VIS measurements of a $50 \mathrm{~nm}$ pentacene film on fused silica, before and after irradiation with $500 \mathrm{eV}$ and $1 \mathrm{keV}$ electrons, shown as dashed black, blue and red curve, respectively. 


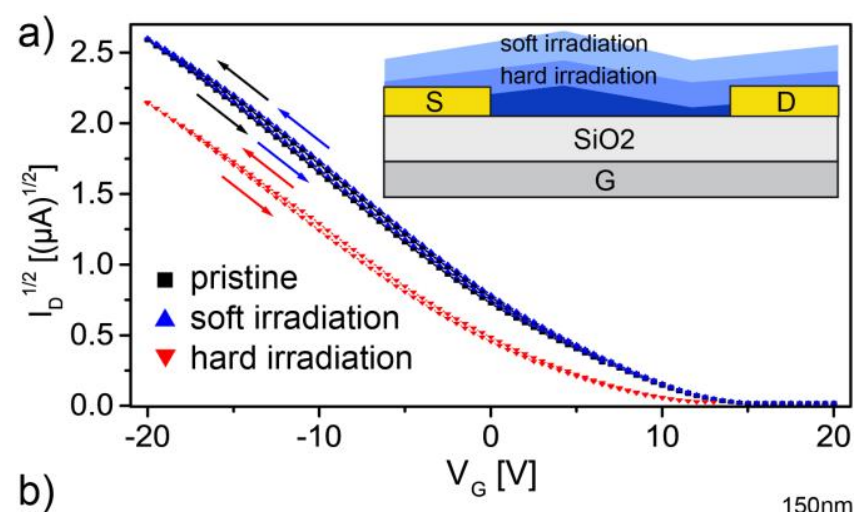

b)
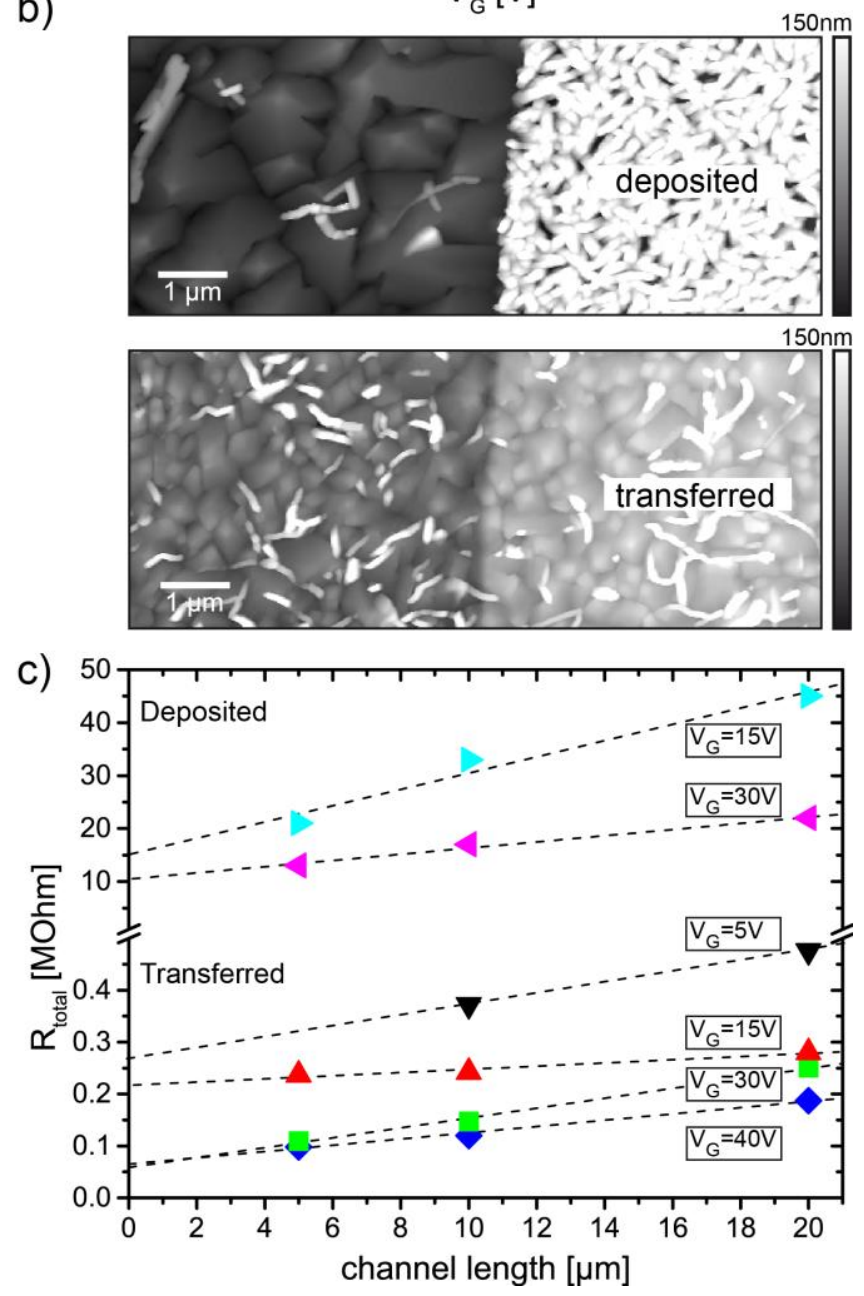

Fig. 4: Characteristic curves of a transistor irradiated at different electron energies and contact resistance analysis of deposited and transferred pentacene on bottom contact transistor geometry. a) Transfer curves of an OFET with pristine, partially crosslinked and strongly crosslinked active layer $\left(\mathrm{V}_{\mathrm{D}}=-10 \mathrm{~V}\right)$. The arrows indicate the sweep direction. Inset: Schematic of the transistor. 
When irradiated softly (low electron energy), only the top layers of the pentacene film are crosslinked, i.e. the conduction channel at the dielectric interface is not affected. After hard irradiation (high electron energy), the crosslinked region penetrates the conduction channel and the device performance is reduced. b) AFM height micrographs of the substrate - contact edge in a transistor channel. Pentacene was vapor deposited (top image), and nanosheet transferred (bottom image). The topography for the transferred film is unchanged on the gold electrode, while the vapor deposited film shows strong dewetting on the contact. The height scale of both AFM images is $150 \mathrm{~nm}$. c) Total channel resistance using vapor deposited pentacene (top) and nanosheet transferred pentacene (bottom), plotted against TFT channel lengths and evaluated for different applied gate voltages. The intersections of the linear fits with the ordinate indicate the extrapolated contact resistances.

\section{Supporting Information}

Detailed information on the experimental procedures, the depth controlled GIXD measurements, crystallinity of transferred pentacene, AFM micrographs of the pentacene surface prior to and after transfer, output characteristics of transferred and deposited TFTs for contact resistance evaluation, helium ion microscopy of mostly crosslinked $20 \mathrm{~nm}$ pentacene film, scanning nearfield optical microscopy of transferred pentacene on gold substrate, and a table listing the specifications of crosslinked samples from main manuscript.

\section{Acknowledgments}

The authors gratefully acknowledge financial support from the Bavarian Ministry for Science through the initiative "Solar Technologies Go Hybrid" (SolTech) as well as by the Deutsche 
Forschungsgemeinschaft (DFG) through the SFB 1032. Parts of this research were carried out at the light source PETRA III at DESY, a member of the Helmholtz Association (HGF). Andrey Turchanin and Nils-Eike Weber acknowledge support by the Deutsche Forschungsgemeinschaft (SPP 1459 „Graphene”) and the EMRP project „GraphOhm“ (The EMRP is jointly funded by the EMRP participating countries within EURAMET and European Union). The research leading to these results has also received funding from the European Union via Graphene Flagship. We thank Dr. Henning Vieker for the help with the acquisition of HIM images. We appreciate time for test measurements at neaspec GmbH. Special thanks to Philipp Altpeter for fruitful discussions and his dedicated technical support in the clean room of the former chair of Prof. Kotthaus (LMU), where many experiments were prepared. 


\section{References}

[1] A. K. Geim, I. V. Grigorieva, Nature 2013, 499, 419.

[2] S. Z. Butler, S. M. Hollen, L. Y. Cao, Y. Cui, J. A. Gupta, H. R. Gutierrez, T. F. Heinz, S. S. Hong, J. X. Huang, A. F. Ismach, E. Johnston-Halperin, M. Kuno, V. V. Plashnitsa, R. D. Robinson, R. S. Ruoff, S. Salahuddin, J. Shan, L. Shi, M. G. Spencer, M. Terrones, W. Windl, J. E. Goldberger, ACS Nano 2013, 7, 2898.

[3] J. W. Suk, A. Kitt, C. W. Magnuson, Y. F. Hao, S. Ahmed, J. H. An, A. K. Swan, B. B. Goldberg, R. S. Ruoff, ACS Nano 2011, 5, 6916.

[4] A. C. Ferrari, F. Bonaccorso, V. Fal'ko, K. S. Novoselov, S. Roche, P. Boggild, S. Borini, F. H. L. Koppens, V. Palermo, N. Pugno, J. A. Garrido, R. Sordan, A. Bianco, L. Ballerini, M. Prato, E. Lidorikis, J. Kivioja, C. Marinelli, T. Ryhanen, A. Morpurgo, J. N. Coleman, V. Nicolosi, L. Colombo, A. Fert, M. Garcia-Hernandez, A. Bachtold, G. F. Schneider, F. Guinea, C. Dekker, M. Barbone, Z. Sun, C. Galiotis, A. N. Grigorenko, G. Konstantatos, A. Kis, M. Katsnelson, L. Vandersypen, A. Loiseau, V. Morandi, D. Neumaier, E. Treossi, V. Pellegrini, M. Polini, A. Tredicucci, G. M. Williams, B. Hee Hong, J.-H. Ahn, J. Min Kim, H. Zirath, B. J. van Wees, H. van der Zant, L. Occhipinti, A. Di Matteo, I. A. Kinloch, T. Seyller, E. Quesnel, X. Feng, K. Teo, N. Rupesinghe, P. Hakonen, S. R. T. Neil, Q. Tannock, T. Lofwander, J. Kinaret, Nanoscale 2015, 7, 4598.

[5] F. Withers, O. Del Pozo-Zamudio, A. Mishchenko, A. P. Rooney, A. Gholinia, K. Watanabe, T. Taniguchi, S. J. Haigh, A. K. Geim, A. I. Tartakovskii, K. S. Novoselov, Nature materials 2015, 14, 301.

[6] B. V. Lotsch, Annual Review of Materials Research 2015, 45, 85.

[7] I. S. Osborne, Science 2016, 353, 458.

[8] D. Jariwala, S. L. Howell, K.-S. Chen, J. Kang, V. K. Sangwan, S. A. Filippone, R. Turrisi, T. J. Marks, L. J. Lauhon, M. C. Hersam, Nano letters 2016, $16,497$.

[9] H. Klauk, Organic Electronics: Materials, Manufacturing, and Applications, WileyVCH, 2006.

[10] E. Menard, V. Podzorov, S. H. Hur, A. Gaur, M. E. Gershenson, J. A. Rogers, Advanced materials 2004, 16, 2097.

[11] A. Turchanin, A. Gölzhäuser, Advanced materials 2016, 28, 6075.

[12] A. Turchanin, D. Kafer, M. El-Desawy, C. Woll, G. Witte, A. Golzhauser, Langmuir 2009, 25, 7342 .

[13] A. Turchanin, A. Beyer, C. T. Nottbohm, X. H. Zhang, R. Stosch, A. Sologubenko, J. Mayer, P. Hinze, T. Weimann, A. Golzhauser, Advanced materials 2009, 21, 1233.

[14] C. T. Nottbohm, A. Turchanin, A. Beyer, R. Stosch, A. Gölzhäuser, Small 2011, 7, 874.

[15] S. Tanuma, C. J. Powell, D. R. Penn, Surf. Interface Anal. 1994, 21, 165.

[16] C.-C. Kuo, T. N. Jackson, Applied Physics Letters 2009, 94, 3304.

[17] T. B. Singh, T. Meghdadi, S. Gunes, N. Marjanovic, G. Horowitz, P. Lang, S. Bauer, N. S. Sariciftci, Advanced materials 2005, 17, 2315.

[18] G. A. Salvatore, N. Munzenrieder, T. Kinkeldei, L. Petti, C. Zysset, I. Strebel, L. Buthe, G. Troster, Nature communications 2014, 5.

[19] S. Schiefer, M. Huth, A. Dobrinevski, B. Nickel, J. Am. Chem. Soc. 2007, 129, 10316.

[20] B. Nickel, R. Barabash, R. Ruiz, N. Koch, A. Kahn, L. C. Feldman, R. F. Haglund, G. Scoles, Physical Review B 2004, 70, 125401.

[21] H. Dosch, B. W. Batterman, D. C. Wack, Physical Review Letters 1986, 56, 1144. 
[22] H. Dosch, Physical Review B 1987, 35, 2137.

[23] G. H. Vineyard, Physical Review B 1982, 26, 4146.

[24] I. K. Robinson, D. J. Tweet, Rep. Prog. Phys. 1992, 55, 599.

[25] C. Westermeier, A. Cernescu, S. Amarie, C. Liewald, F. Keilmann, B. Nickel, Nature communications 2014, 5, 4101.

[26] N. D. Baydoğan, Materials Science and Engineering: B 2004, 107, 70.

[27] C. Rost, D. J. Gundlach, S. Karg, W. Riess, Journal of Applied Physics 2004, 95, 5782.

[28] D. Käfer, L. Ruppel, G. Witte, Physical Review B 2007, 75, 085309.

[29] K. Hong, S. Y. Yang, C. Yang, S. H. Kim, D. Choi, C. E. Park, Organic electronics 2008, 9,864 .

[30] Y. Tsuruma, A. Al-Mahboob, S. Ikeda, J. T. Sadowski, G. Yoshikawa, Y. Fujikawa, T. Sakurai, K. Saiki, Advanced materials 2009, 21, 4996.

[31] W. S. Hu, Y. T. Tao, Y. J. Hsu, D. H. Wei, Y. S. Wu, Langmuir 2005, 21, 2260.

[32] H. Kim, Z. Meihui, N. Battaglini, P. Lang, G. Horowitz, Organic electronics 2013, 14, 2108.

[33] H. Yanagisawa, T. Tamaki, M. Nakamura, K. Kudo, Thin Solid Films 2004, 464, 398.

[34] D. J. Gundlach, L. Zhou, J. A. Nichols, T. N. Jackson, P. V. Necliudov, M. S. Shur, Journal of Applied Physics 2006, 100, 024509.

[35] G. Horowitz, P. Lang, M. Mottaghi, H. Aubin, Advanced Functional Materials 2004, 14, 1069. 